\title{
A Corpus-Based Study on Construction of "Anger Adjectives + Prepositions" in World Englishes
}

\author{
Xinlu Zhang ${ }^{1} \&$ Jingxiang Cao ${ }^{1}$ \\ ${ }^{1}$ School of Foreign Languages, Dalian University of Technology, Dalian, China \\ Correspondence: Jingxiang Cao, School of Foreign Languages, Dalian University of Technology, Dalian, \\ Liaoning, 116024, China. E-mail: caojx@dlut.edu.cn
}

Received: June 22, 2021

Accepted: July 29, $2021 \quad$ Online Published: August 1, 2021

doi:10.5539/ells.v11n3p55

URL: https://doi.org/10.5539/ells.v11n3p55

\begin{abstract}
Anger as one of the basic emotions has attracted much attention. In the construction of "Anger adjectives + prepositions", the temporal duration of the Anger adjectives is closely related to their prepositional collocates. Differences in the use of the Anger adjectives and their prepositional collocates might be captured in the world English varieties. The corpora used in this study cover eight varieties of English. The five varieties of English used in Canada, Philippines, Singapore, India and Nigeria are from the International Corpus of English (ICE). The China English corpus (ChiE) consists of news texts crawled from six Chinese English media. American English is taken from the Corpus of Contemporary American English (COCA) and British English is taken from British National Corpus (BNC). By investigating the use of the Anger adjectives and their prepositional collocates in the eight varieties of English, this paper finds that, on the continuums of the temporal duration of Anger adjectives, most varieties of English are closer to American English, whereas only Singapore English is close to British English. The distribution of Anger adjectives in the English varieties is largely in accordance with the Concentric Circles of world Englishes whereas the continuums of the temporal duration of emotions present a new insight into their relations.
\end{abstract}

Keywords: anger adjectives, prepositions, world Englishes

\section{Introduction}

Scientists have predicted that the 21 st century will be the century for emotions. It is argued that the use of emotional words can reflect cultural differences and they vary across languages and cultures (Wierzbicka, 1999, p. 34). Fontaine and Scherer (2013, p. 134) have argued that an emotional word has the component of duration, indicating how long the emotion lasts. By investigating the temporal duration of emotion clusters, they found out that among the emotion clusters, the anger cluster lasts relatively shorter than fear, sadness and joy. Prepositions serve an important role when collocating with emotional words. Scholars such as Kim Eunmi have claimed that there is a close relationship between the prepositional collocates of emotional adjectives and the temporal duration of the emotions they denote. In recent years, English is discussed in the global context. Differences in the use of the Anger adjectives and their prepositional collocates in the different varieties of English are expected to be captured to show cultural differences.

Therefore, enlightened by the identification of temporal duration of emotions in Fontaine and Scherer (2013, pp. $142-145)$ and the researches on the collocation principle of emotional adjectives and prepositions in Kim (2015, 2016, 2018), this paper will explore the construction of "Anger adjectives + prepositions" in eight varieties of English, attempting to verify the relationship between varieties of English in terms of this construction, and thus to present a new insight of the relationship between them. This paper intends to answer the following research questions:

a) What's the distribution of Anger adjectives in the English varieties?

b) Is there any difference in the prepositional collocates of Anger adjectives in the English varieties?

c) Is there any difference in the continuums of the temporal duration of Anger adjectives indicated by their prepositional collocates in the English varieties? 


\section{Literature Review}

\subsection{Construction of "Anger Adjectives + Prepositions"}

\subsubsection{Emotion of Anger}

Anger, as one of the most often discussed emotion concepts, has been defined as an intense, bitter, and sometimes violent emotion (Sorial, 2017, p. 123). Among most of the known classification of emotions (e.g., Oatley \& Johnson-Laird, 1987, p. 33; Fontaine et al., 2013, p. 87), anger belongs to one of the basic emotions including anger, fear, sadness, joy, etc., which are universal and biologically rooted across cultures and languages. Anger is a basic-level and prototypical emotion category (Kövecses, 2000, p. 5). Emotions, including anger, can be defined as dynamic episodes of subsystem synchronization and are thus extended in time (Fontaine $\&$ Scherer, 2013, p. 134). The temporal duration is an essential aspect of emotions. The temporal duration of emotional nouns in Anger cluster has been tested relatively shorter than that in Joy, Sadness and Fear clusters in a study of the duration of the 24 basic emotion nouns as well as that of the four emotion clusters (Fontaine \& Scherer, 2013, p. 108). A significant difference within the Anger cluster has been found: jealousy, hate, and contempt have significantly longer durations than anger and irritation. Similar results are found in a more recent investigation that verifies the close relationship between the temporal duration of emotions and their prepositional collocates (Kim, 2018, pp. 237-240). The study also confirms the relatively shorter temporal duration of emotional adjectives included in the Anger cluster by focusing on the collocation of emotional adjectives and prepositions.

The inextricable relationship among emotion, language and culture has been confirmed by several scholars. Cognitive linguists stress the importance of variety of language in the study of emotions. That is because "whatever the conditions that produce an emotion like anger, whether or not it is visibly expressed, and whatever physiological responses accompany it, it is only through language that we can know that what is experienced is anger" (Wierzbicka \& Harkins, 2001, pp. 2-3). Additionally, it has been argued that social and cultural factors play important roles in the study of emotions. Kövecses (2000, p. 159) also argues that "anger is a social construction and thus varies considerably from culture to culture". And it is worth noting that cognitive linguistics provides many important perspectives to analyze emotions, with prototypical theory being one of the most crucial perspectives. In general, "linguistic categories may be fuzzy at the edges but clear in the centre" (Geeraerts, 2010, p. 183). According to Izdebska (2015, p. 18), words in the same category being used will result in differences, which are not only in the scale of the emotion but also in the prototypical scenario, evaluation and consequences. Among the Anger adjectives which will be discussed in the construction of "Anger adjectives + prepositions" in this paper, angry will be regarded as the prototypical representative of the category, whereas furious might be more peripheral. In this paper, not only the comparison of such construction in the English varieties will be conducted, but also the Anger adjectives within each variety of English will be compared.

\subsubsection{Prepositions in the Construction of "Anger Adjectives + Prepositions"}

Prepositions serve an important role when collocating with emotional words, especially because the selection of prepositions has a close relationship with the temporal duration of emotions.

According to Osmond (1997, p. 112), there are eight prepositions, namely, by, with, at, about, of, to, for, and over, being widely used as the collocates of emotional words. Bolinger (1984, p. 55) classifies emotions into two groups, namely, emotion caused and emotion projected. In accordance with this classification, emotional words, not only emotion nouns but also emotional adjectives, that collocate with the prepositions for and of are categorized as projective emotions because these two prepositions have a strong tendency to occur with emotional words describing long-term emotions, which is supported by Kim $(2015$, pp. 118, 132) as well. Dirven (1995, p. 98) classifies prepositions into three sets, namely, proximity prepositions (at, with, by), source prepositions (of, from, out of), and area or volume prepositions (in, about, over), in order to present a systematic analysis of expressions of cause in English. Dirven's study confirms the close relationship between over and about with some synchronic examples, "replace over with about" and "a satisfying result" can also be obtained. Similarly, Ku (2019, p. 11) argues that an emotional adjective combining with over refers to the event extending over the whole process that arouses one's emotion. A more recent study by Kim (2018) provides the definition of four prepositions, namely, at, by, with, about, used in the collocation of emotional adjectives and prepositions based on their source meaning and she classifies the four prepositions into different dimensions in terms of the dimensional typology of Rhee's as well as the source spatial meaning of prepositions. Kim (2018, p. 234) argues that prepositions in dimension 0 and 1 tend to collocate with short-term emotions while prepositions in dimension 2 and 3 tend to collocate with long-term emotions.

Therefore, indicated by prepositional collocates, emotions in the construction of "Anger adjectives + 
prepositions" can be classified into two types. There are two prepositions, at and by, which tend to collocate with short-term emotions. There are five prepositions, namely, for, of, with, about, and over, which tend to collocate with long-term emotions.

\subsection{World Englishes}

According to Firth (1956, p. 97), English should be "an international language in the Commonwealth, the Colonies and in America" and "it is international not only in Europe but in Asia and Africa", and thus it serves various ways of people's life in different cultural environments.

For the spread and development of world Englishes, several models of English as a global language have been conducted, among which the most influential one is the Concentric Circles proposed by Kachru in 1985. It can be regarded as a theoretical framework to clearly display "the pluricentricity in English and its speakers around the world" (Karakaş, 2017, p. 111) and it can provide the schema for the conceptualization of varieties of English. This framework consists of three circles: the Inner Circle, the Outer Circle and the Expanding Circle. Places included in the Inner Circle are those long-established bases of English (e.g., UK, USA, and Canada) where English is primarily spoken as a mother tongue by native speakers. Places in the Outer Circle (e.g., India, and Singapore) refer to those where English functions as the second language that is institutionalized as an additional language and is thus often used for communication within the region. As for places belonging to the Expanding Circle (e.g., China), English has not been institutionalized yet and it is regarded as a foreign language, thus mainly used for international communication as well as language taught in classroom. This paper attempts to provide a new insight about the relations of varieties of English in terms of the construction of "Anger adjectives + prepositions".

\subsection{Research Gap}

Emotions have been studied from various perspectives such as cross-culture perspective, psycholinguistics, sociolinguistics, etc., while few studies on the collocation of "emotional adjectives + prepositions" can be found. Among the very few studies focusing on the collocation of emotional words and prepositions, Dirven (1995, 1997) only focuses on the emotions expressed by prepositional phrases and Osmond (1997) fails to explain the relationship between the temporal duration of emotions and prepositions. More recently, Kim $(2015,2018)$ and $\mathrm{Ku}(2019)$ investigates the collocation patterns of emotional adjectives with prepositions in semantics and pragmatics respectively, which are notable examples and provide inspiration for this study. However, their studies only focus on collocation of "emotional adjectives + prepositions" based on data from American English but fail to explore such patterns in a more global environment and thus fail to unveil the possible differences of such patterns that might be explained by cultural differences. In terms of the few studies of anger in world Englishes, some aim to uncover differences in the process of expressing this emotion in different situations for learners of multiple languages (Dewaele \& Qaddourah, 2016), others aim to explore causes and characteristics of the emotion of anger in particular situations and thus to confirm the close relationship between emotion, language and society (Van Kleef \& Côté, 2007), but no studies focus on the construction of "Anger adjectives + prepositions".

\section{Methodology}

The corpora used in this paper cover eight varieties of English. Five varieties, namely, English in Canada (CanE), Philippines (PhiE), Singapore (SinE), India (IndE) and Nigeria (NigE), are collected from five regional components of the International Corpus of English (ICE; Nelson, 1996). China English corpus (ChiE) consists of written news crawled from China Daily, Xin Hua News, The State Council, The Ministry of Foreign Affairs, China Today, and Beijing Weekend, which are all generally regarded a high level of China English (Li, 2016, p. 31). American English (AmE) from the Corpus of Contemporary American English (COCA) and British English (BriE) from British National Corpus (BNC) are also included in the study. Table 1 gives the size of the corpora.

Table 1. List of corpora

\begin{tabular}{lll}
\hline Name & Variety & Size (Tokens) \\
\hline AmE & America English & 1001610938 \\
BriE & British English & 96263399 \\
ChiE & China English & 46715888 \\
IndE & India English & 1639757 \\
PhiE & Philippines English & 1600502 \\
CanE & Canada English & 1593935 \\
SinE & Singapore English & 1550257 \\
NigE & Nigeria English & 1022649 \\
\hline
\end{tabular}


As the combination of Fontaine et al. (2013, p. 88) and Kim (2018, p. 228), twenty Anger adjectives are selected, namely, angry, angrier, angriest, mad, madder, maddest, outraged, annoyed, riley, vexed, contemptuous, scornful, sniffish, indignant, peeved, irritated, furious, hateful, jealous, and blanky.

AntConc, Tree Tagger and Microsoft Excel are used in the data analysis. AntConc, a freeware corpus analysis toolkit developed by Anthony (2002), was used to collect data from corpora, including wordlists, prepositional collocates and concordances of Anger adjectives. Tree Tagger (Schmid, 1994) was used to POS tag the corpora. Microsoft Excel was used for data analysis.

The data processing of this paper is as follows. Tree Tagger was used to tag words by part of speech. In such a way, words, together with their part of speech that is needed were searched thus getting rid of the unwanted cases. Then AntConc was used to obtain the frequency list of the Anger adjectives in each corpus, and thus their distribution in the English varieties were compared. The prepositional collocates of the Anger adjectives in all corpora were investigated as well to compare their temporal duration in the English varieties. Based on the classification of emotions of this paper, the continuums of the temporal duration of the Anger adjectives indicated by their prepositional collocates were presented and thus comparisons of the construction of "Anger adjectives + prepositions" in world Englishes were conducted. Additionally, it is worth noting that prepositional collocates that are not necessarily related to the arousal of the emotion being discussed, such as mad with frustration and furious in a famous interview have been excluded. In addition, only prepositional collocates of Anger adjectives that have MI's of over 3.0 are selected in this paper because typically scores of approximately 3.0 or above show semantic bonding between the two words.

\section{Results and Discussion}

\subsection{Distribution of Anger Adjectives in the English Varieties}

A total of 20 Anger adjectives were selected as mentioned in Section 3, and the standardized frequency of the Anger adjectives in the English varieties is shown in Figure 1.

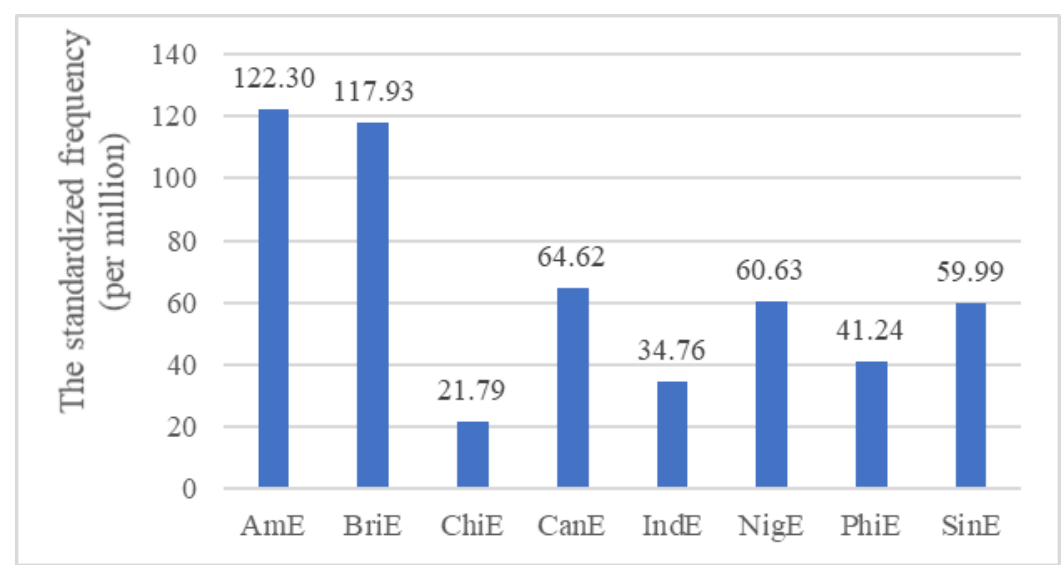

Figure 1. The standardized frequency of the Anger adjectives in the English varieties

As Figure 1 shows, there is a sharp contrast in the use of the Anger adjectives among different varieties of English. To be specific, the Anger adjectives are used more frequently in places including America and Britain where English is regarded as the dominant and official language, whereas they are used very infrequently in places like China where English is only regarded as a foreign language and used only in particular situations such as international meetings, classroom, etc. Even in those places like Singapore where English is regarded as a second language and used relatively frequently in people's daily life, the Anger adjectives are used obviously less frequently than places like America and Britain. That can be roughly explained that, compared with native speakers, people in places where English is seen as a foreign language are relatively more restricted in the use of emotional adjectives expressing the emotion of anger. The distribution of the Anger adjectives in the English varieties is largely in accordance with the Three Concentric Circles of World Englishes (Kachru, 2006, p. 196). Taking ChiE as an example, the restriction of the use of the Anger adjectives has been confirmed not only in this study but also in previous studies (e.g., Dewaele \& Pavlenko, 2002; Rong, 2007, etc.). 


\subsection{Prepositional Collocates of Anger Adjectives in the English Varieties}

In this process, only Anger adjectives that actually occur with prepositional collocates in all the corpora are discussed. In order to select those Anger adjectives in all the corpora, we also investigate the number of types of prepositional collocates of all the Anger adjectives in each corpus as shown in Table 2.

Table 2. The number of types of prepositional collocates of the Anger adjectives in the English varieties

\begin{tabular}{lllllllll}
\hline & AmE & BriE & ChiE & IndE & PhiE & CanE & SinE & NigE \\
\hline angry & 3 & 3 & 5 & 1 & 2 & 3 & 1 & 1 \\
annoyed & 3 & 4 & 1 & 0 & 2 & 2 & 3 & 1 \\
blanky & 0 & 0 & 0 & 0 & 0 & 0 & 0 & 0 \\
contemptuous & 1 & 0 & 0 & 0 & 0 & 0 & 0 & 0 \\
furious & 3 & 3 & 4 & 0 & 0 & 0 & 0 & 0 \\
hateful & 0 & 0 & 0 & 0 & 0 & 0 & 0 & 0 \\
indignant & 3 & 2 & 3 & 0 & 0 & 0 & 1 & 0 \\
irritated & 3 & 1 & 1 & 1 & 0 & 1 & 1 & 0 \\
jealous & 1 & 1 & 1 & 0 & 1 & 1 & 1 & 1 \\
mad & 2 & 1 & 1 & 0 & 2 & 2 & 1 & 0 \\
outraged & 4 & 2 & 2 & 0 & 0 & 0 & 0 & 0 \\
peeved & 3 & 2 & 0 & 1 & 0 & 1 & 0 & 0 \\
riley & 0 & 0 & 0 & 0 & 0 & 0 & 0 & 0 \\
scornful & 1 & 0 & 0 & 0 & 0 & 0 & 0 & 0 \\
sniffish & 0 & 0 & 0 & 0 & 0 & 0 & 0 & 0 \\
vexed & 1 & 2 & 2 & 0 & 0 & 0 & 0 & 0 \\
\hline
\end{tabular}

Note. Anger adjectives in their comparative and superlative forms are also included.

As Table 2 shows, only very few Anger adjectives have prepositional collocates in all the corpora. Accordingly, four Anger adjectives, namely, angry, annoyed, jealous, and mad are selected for the following analysis. Table 2 shows that the number of types of prepositional collocates of the Anger adjectives varies across corpora, except for that of jealous with very limited number of types of prepositional collocates in all corpora. The types of prepositional collocates of the Anger adjectives in IndE and NigE are very limited. Only the prepositional collocates of angry in IndE and NigE and that of annoyed and jealous in NigE can be found. The types of the Anger adjectives used in the two corpora are less varied than the other corpora. As for ChiE, however, the infrequent use of the Anger adjectives in this corpus, as shown in Figure 1, does not result in a limited number of types of prepositional collocates of the Anger adjectives. Instead, the prepositional collocates of the Anger adjectives in ChiE varied most. Types of prepositional collocates of the Anger adjectives in IndE and NigE are very limited although the proportion of the Anger adjectives in the two corpora are higher than that of ChiE. Therefore, it can be roughly summarized that, in terms of the number of types of prepositional collocates of the Anger adjectives, ChiE has the largest variety while IndE and NigE have the smallest, indicating that Chinese people tend to choose more prepositions collocating with the Anger adjectives when expressing the emotion of anger but the opposite is true for Indians and Nigerians. For Indians and Nigerians, more emotional adjectives are used to express anger but these adjectives do not often have a prepositional collocate and often have a rigid choice for their prepositional collocates.

Tables 3-6 present the prepositional collocates of the four major Anger adjectives in the varieties in detail.

Table 3. Prepositional collocates of angry and the frequency of collocation in the varieties

\begin{tabular}{llllllll}
\hline Corpus & angry & at & by & with & about & over & of \\
\hline AmE & 52980 & 4435 & 0 & 2769 & 1906 & 0 & 0 \\
BriE & 4000 & 0 & 3 & 388 & 99 & 0 & 0 \\
ChiE & 478 & 35 & 0 & 22 & 21 & 19 & 1 \\
IndE & 20 & 0 & 0 & 3 & 0 & 0 & 0 \\
PhiE & 16 & 1 & 0 & 1 & 0 & 0 & 0 \\
CanE & 39 & 7 & 0 & 2 & 4 & 0 & 0 \\
SinE & 34 & 0 & 0 & 6 & 0 & 0 & 0 \\
NigE & 21 & 0 & 0 & 2 & 0 & 0 & 0 \\
\hline
\end{tabular}


Table 4. Prepositional collocates of annoyed and the frequency of collocation in the varieties

\begin{tabular}{llllll}
\hline Corpus & annoyed & at & by & with & about \\
\hline AmE & 4929 & 524 & 716 & 538 & 0 \\
BriE & 870 & 68 & 61 & 80 & 37 \\
ChiE & 63 & 0 & 13 & 0 & 0 \\
IndE & 7 & 0 & 0 & 0 & 0 \\
PhiE & 7 & 0 & 1 & 2 & 0 \\
CanE & 5 & 1 & 0 & 2 & 0 \\
SinE & 8 & 1 & 0 & 2 & 2 \\
NigE & 7 & 0 & 0 & 2 & 0 \\
\hline
\end{tabular}

Table 5. Prepositional collocates of jealous and the frequency of collocation in the varieties

\begin{tabular}{llll}
\hline Corpus & jealous & of & about \\
\hline AmE & 12285 & 2842 & 0 \\
BriE & 897 & 260 & 0 \\
ChiE & 42 & 21 & 0 \\
IndE & 4 & 0 & 0 \\
PhiE & 8 & 3 & 0 \\
CanE & 10 & 3 & 0 \\
SinE & 9 & 2 & 1 \\
NigE & 8 & 4 & 0 \\
\hline
\end{tabular}

Table 6. Prepositional collocates of $\mathrm{mad}$ and the frequency of collocation in the varieties

\begin{tabular}{llll}
\hline Corpus & mad & at & about \\
\hline AmE & 40286 & 7815 & 1295 \\
BriE & 2986 & 0 & 50 \\
ChiE & 104 & 4 & 0 \\
IndE & 11 & 0 & 0 \\
PhiE & 25 & 5 & 2 \\
CanE & 36 & 10 & 1 \\
SinE & 26 & 0 & 2 \\
NigE & 18 & 0 & 0 \\
\hline
\end{tabular}

As Tables 3-6 show, among all the corpora, prepositional collocates of the Anger adjectives vary across words. For example, for prepositional collocates of annoyed, as shown in Table 4, with having the tendency of collocating with long-term emotions is its only prepositional collocate in NigE but by which tends to collocate with short-term emotions is its only collocate in ChiE. Additionally, in AmE, BriE, CanE, PhiE, and SinE, prepositional collocates of annoyed, as shown in Table 4, from both long-term and short-term prepositions can be found. One intriguing aspect to note here is that of is the main prepositional collocate of jealous in all the corpora as shown in Table 5.

On the other hand, some similarities among some corpora can be found. For AmE, CanE, and PhiE, the types of prepositional collocates of the Anger adjectives are similar. To be specific, prepositions with the tendency of collocating with both long-term and short-term emotions collocate with all the Anger adjectives, except for jealous, in all these corpora. For BriE, SinE, IndE and NigE, prepositions with the tendency of collocating with long-term emotions are the main prepositional collocates of nearly all the Anger adjectives, except for annoyed in BriE collocating with prepositions with the tendency of collocating with both long-term and short-term emotions and having roughly the same frequency of collocation, as shown in Table 4.

For ChiE, the situation is more complicated. As Tables 4 and 6 show, annoyed and mad in ChiE only collocates with the preposition with the tendency of collocating with short-term emotions. But the diversified characteristic of types of prepositional collocates of the Anger adjectives in ChiE is reflected when exploring prepositional collocates of angry, as shown in Table 3. More specifically, five types of prepositional collocates of angry are found. Among these prepositional collocates, of and over collocating with angry are not found in other corpora. That is not in accordance with the results of $\mathrm{Ku}$ (2019), who confirms the restriction of selection of prepositions when they are used to collocate with emotional adjectives. The disagreement may be caused by the difference in the corpora used in the two studies. Based on materials provided by the Korean high school textbooks, what Ku 
(2019) actually discovers is the characteristic of prepositional collocates of emotional adjectives in Korean high school textbooks, and that result cannot represent the characteristic of prepositional collocates of emotional adjectives in cultural environments where English is regarded as a foreign language. However, the materials included in ChiE in this paper are all generally regarded a high level of China English. Previous studies (e.g., Dewaele \& Pavlenko, 2002; Woon, 2009; Dewaele \& Qaddourah, 2016, etc.) have suggested that language competence influences the emotional expressions of bilinguals. The diversity in the use of prepositional collocates of the Anger adjectives in ChiE may also be explained by the effect of language proficiency on the use of emotional words in spite of the limited proportion of the Anger adjectives in this corpus. Therefore, this paper also confirms that the use of prepositions collocating with emotional adjectives by English learners with a relatively high-level shows diversity.

\subsection{Temporal Duration of the Anger Adjectives Indicated by Their Prepositional Collocates in the English Varieties}

Emotional adjectives collocating with different prepositions tend to express emotions with different temporal duration. The temporal duration of Anger adjectives indicated by their prepositional collocates can be explored based on Tables 3-6. However, due to the complicated collocation of preposition with the Anger adjectives in the varieties, the percentage of the long/short prepositional collocates of Anger adjectives within each corpus has been investigated as well, as shown in Table 7.

Table 7. The long/short prepositional collocates of the Anger adjectives in the varieties

\begin{tabular}{|c|c|c|c|c|c|c|c|c|c|}
\hline \multirow[t]{2}{*}{ Corpus } & \multirow[t]{2}{*}{ prep. } & \multicolumn{2}{|l|}{ angry } & \multicolumn{2}{|c|}{ annoyed } & \multicolumn{2}{|c|}{ jealous } & \multicolumn{2}{|l|}{ mad } \\
\hline & & freq. & $\%$ & freq. & $\%$ & freq. & $\%$ & freq. & $\%$ \\
\hline \multirow[t]{2}{*}{$\mathrm{AmE}$} & Long & 4675 & 51.32 & 538 & 30.25 & 2842 & 100.00 & 1295 & 14.22 \\
\hline & Short & 4435 & 48.68 & 1240 & 69.74 & 0 & 0.00 & 7815 & 85.78 \\
\hline \multirow[t]{2}{*}{ BriE } & Long & 487 & 99.39 & 117 & 47.56 & 260 & 100.00 & 50 & 100.00 \\
\hline & Short & 3 & 0.61 & 129 & 52.44 & 0 & 0.00 & 0 & 0.00 \\
\hline \multirow[t]{2}{*}{ ChiE } & Long & 63 & 64.29 & 0 & 0.00 & 21 & 100.00 & 0 & 0.00 \\
\hline & Short & 35 & 35.71 & 13 & 100.00 & 0 & 0.00 & 4 & 100.00 \\
\hline \multirow[t]{2}{*}{ IndE } & Long & 3 & 100.00 & 0 & 0.00 & 0 & 0.00 & 0 & 0.00 \\
\hline & Short & 0 & 0.00 & 0 & 0.00 & 0 & 0.00 & 0 & 0.00 \\
\hline \multirow[t]{2}{*}{ PhiE } & Long & 1 & 50.00 & 2 & 66.67 & 3 & 100.00 & 2 & 28.57 \\
\hline & Short & 1 & 50.00 & 1 & 33.33 & 0 & 0.00 & 5 & 71.43 \\
\hline \multirow[t]{2}{*}{ CanE } & Long & 6 & 46.15 & 2 & 66.67 & 3 & 100.00 & 1 & 9.10 \\
\hline & Short & 7 & 53.85 & 1 & 33.33 & 0 & 0.00 & 10 & 90.91 \\
\hline \multirow[t]{2}{*}{$\operatorname{Sin} E$} & Long & 6 & 100.00 & 4 & 80.00 & 3 & 100.00 & 2 & 100.00 \\
\hline & Short & 0 & 0.00 & 1 & 20.00 & 0 & 0.00 & 0 & 0.00 \\
\hline \multirow[t]{2}{*}{ NigE } & Long & 2 & 100.00 & 2 & 100.00 & 4 & 100.00 & 0 & 0.00 \\
\hline & Short & 0 & 0.00 & 0 & 0.00 & 0 & 0.00 & 0 & 0.00 \\
\hline
\end{tabular}

In Table 7, freq. indicates the frequency of all the prepositional collocates of each adjective and the percent indicates the proportion of the collocation with long or short-term group prepositions to the total frequency of collocation with all prepositions. Therefore, the continuums of the temporal duration of Anger adjectives in the varieties are presented based on the proportion of the collocations with long-term group prepositions:

AmE, ChiE, IndE, and NigE: jealous $>$ angry $>$ annoyed $>$ mad;

CanE and PhiE: jealous $>$ annoyed $>$ angry $>$ mad;

BriE and SinE: jealous $>$ mad $>$ angry $>$ annoyed

It can be seen that AmE and BriE have different continuums of the temporal duration of the Anger adjectives. For the other varieties, most of them are closer to AmE except for SinE, which is closer to BriE. Albeit with the slight difference in CanE and PhiE where the temporal duration of annoyed is relatively longer than that of angry, the continuums of the temporal duration of the Anger adjectives for varieties except for BriE and SinE are similar and it is largely in agreement with Fontaine and Sherer (2013) and Kim (2018). Fontaine and Sherer (2013), from a psychological perspective, examine the differences in temporal duration of emotions within the same emotion cluster as well as among different clusters. Within the Anger cluster, Fontaine and Sherer (2013, pp. 142-145) argue that the temporal duration of jealousy is longer than anger. Similarly, Kim (2018, p. 248), focusing on the collocation principle of prepositions with emotional adjectives based on data from COCA, 
confirms the relatively longer duration of jealous and the relatively shorter duration of mad, with angry and annoyed in between.

On the other hand, the continuums of the temporal duration of the Anger adjectives in BriE and SinE are similar but different from those of the other corpora. That is because, the Anger adjectives in these two corpora mainly collocate with prepositions having the tendency of collocating with long-term emotions, such as, with, about and of, while in other corpora the Anger adjectives mainly collocate with prepositions with the tendency of collocating with short-term emotions or those with the tendency of collocating with both long-term and short-term emotions. In the two corpora, nearly all the prepositional collocates of angry, mad and jealous belong to long-term prepositions, but that of angry and mad in most of the other corpora in this paper are both long-term and short-term prepositions. The following examples are expected to help distinguish the different temporal duration of the same Anger adjective when it collocates with different groups of prepositions.

(a) Nancy Pelosi is arrogant. I sat down and wrote her a letter Friday, I was so angry at her and her co-horts. GOOD for you guys, GOP! (COCA)

(b) While I am angry with this administration for four years of failure and lies, I am even more angry with the liberal party this election. (COCA)

(c) Mr Wolski was suddenly angry at the Zoo for keeping such creatures in such a way.

(d) I think the Algerians have been angry with him for some time and are prepared to let Polisario have a free hand. (BNC)

(e) Shouldn't be hard, Fanning thought absently. Venera's not mad at me for anything at the moment. He risked another glance at the battle. (COCA)

(f) Constanza was madabout horses during her first twenty years. She had complete physical courage.

(BNC)

In example (a), the emotion of anger is expressed to somebody after a series of momentary actions "sit down" and "write a letter" and the exclamation mark at the end of the sentence shows that the emotion of the speaker when uttering this sentence is intense and short and thus the temporal duration of anger here collocating with at is short. More obviously, in example (c), the use of the adverb "suddenly" helps to denote a sudden arousal of the emotion of anger. Kim (2018) investigates the collocation pattern of prepositions with the adverb suddenly and found that the adverb suddenly collocates with prepositions having the tendency of collocating with short-term emotions more frequently than prepositions having the tendency of collocating with long-term emotions. By contrast, in examples (b) and (d), the emotion of anger is longer because it can last for some time and has continued for a while. Therefore, it can be summarized that the temporal duration of the same emotional adjective, angry here, is relatively shorter when it collocates with preposition at than with. For mad in example (e), the adverbial of time shows that the temporal duration of the emotion of madness is short because it only occurs "at the moment" of the speaking. By contrast, the temporal duration of mad in example (f) is relatively longer because it has continued for "twenty years". Therefore, it can be summarized that the temporal duration of the same Anger adjective also differs with respect to its prepositional collocates.

The similarity on the temporal duration of the Anger adjectives indicated by their prepositional collocates as well as their prepositional collocates between BriE and SinE might be explained by the effect of culture and society on emotional words. The close relationship between the two can be traced. Singapore was once a colony of England. From the British Raffles landed in Singapore in 1819 to Singapore established a self-governing state in 1963, the British colonized Singapore for more than 140 years. What's more, this study also speculates that the difference among BriE, SinE and other corpora might be caused by the setting of MI score, so the MI score is adjusted specially for the two corpora. For BriE, when the MI score of the selected prepositional collocates of the Anger adjectives is set to above 1.0, different results are found. More specifically, the temporal duration of the Anger adjectives indicated by their prepositional collocates as well as their prepositional collocates thus show a new situation which is similar to those in other corpora like AmE and ChiE in this study. However, for SinE, the temporal duration of the Anger adjectives indicated by their prepositional collocates as well as their prepositional collocates are still different from other corpora albeit with relatively low MI score of prepositions.

\section{Conclusion}

This paper conducts the comparison of the construction of "Anger adjectives + prepositions" in eight varieties of English. The Anger adjectives used in this paper are selected as the combination of Fontaine et al. $(2013$, p. 88) and Kim (2018, p. 228). Distribution of the Anger adjectives in the English varieties is investigated and it is found that this distribution is largely in accordance with the Concentric Circles of world Englishes (Kachru, 
2006, p. 196). More specifically, for places included in the Inner Circle, including America and Britain, the Anger adjectives are used more frequently than places included in Outer Circle of English, such as Singapore and Canada, followed by places included in the Expanding Circle of English such as China. Based on the assumption that emotional adjectives collocating with different prepositions tend to express different durations of emotions, this paper investigates the prepositional collocates of the Anger adjectives in the English varieties, so that the temporal duration of emotions has been explored. It is found that, in terms of the continuums of the temporal duration of Anger adjectives, most varieties of English are closer to American English, whereas only Singapore English is close to British English. Results of the temporal duration of the Anger adjectives in varieties of English including AmE are supported by Fontaine and Sherer (2013, pp. 142-145) and Kim (2018, p. 248). More specifically, this paper also confirms the relatively longer duration of jealous and the relatively shorter duration of mad, with angry and annoyed in between.

Many scholars argue that language and culture are inseparable when talking about emotions. But there are no related studies on the construction of "Anger adjectives + prepositions" in world Englishes. In addition, it is necessary to uncover potential cultural differences persist among people who vary in their cultural orientations when they use the same language (Tsai et al., 2004, p. 1227). Therefore, this paper is significant for providing a comparison of such a construction in the English varieties.

However, this paper has not yet found obvious cultural differences in term of this construction, which might be caused by the limitation of collected data. In the future, the investigation of a larger set of varieties is of course possible. In addition to the construction of "Anger adjectives + prepositions" investigated in this paper, other emotional words, especially Anger nouns is in need for further study.

\section{Acknowledgements}

The study is supported by the Project (No.18YJA740002) of the Foundation for Humanities and Social Sciences of the Ministry of Education, China.

\section{References}

Anthony, L. (2019). AntConc (Version 3.5.8). Tokyo, Japan: Waseda University. Retrieved from https://www.laurenceanthony.net/software

Bolinger, D. (1984). Surprise. In L. J. Raphael, C. B. Raphael \& M. R. Valdovinos (Eds.), Language and Cognition. Cognition and Language: A Series in Psycholinguistics (pp. 45-58). Springer, Boston, MA. https://doi.org/10.1007/978-1-4899-0381-5_5

Dewaele, J. M., \& Pavlenko, A. (2002). Emotion Vocabulary in Interlanguage. Language Learning, 52(2), 263-322. https://doi.org/10.1111/0023-8333.00185

Dewaele, J. M., \& Qaddourah, I. (2016). Language choice in expressing anger among Arab-English Londoners. Russian Journal of Linguistics (Vestnik Rudn), 23(4), 82-100.

Dirven, R. (1995). The construal of cause: The case of cause prepositions. Language and the Cognitive Construal of the World, 9c1(2), 95-118. https://doi.org/10.1515/9783110809305.95

Dirven, R. (1997). Emotions as cause and the cause of emotions. In S. Niemeier \& R. Dirven (Eds.), The language of emotions: Conceptualization, expression, and theoretical foundation (pp. 55-83). Amsterdam: John Benjamins. https://doi.org/10.1075/z.85.06dir

Firth, J. R. (1956). Descriptive linguistics and the study of English. In R. P. Frank (Ed.), Selected Papers of J.R. Firth (1952-1959) (pp. 96-113). London and Bloomington: Longman and Indiana University Press.

Fontaine, J. R., \& Scherer, K. R. (2013a). The global meaning structure of the emotion domain: Investigating the complementarity of multiple perspectives on meaning. In J. R. Fontaine, K. R. Scherer \& C. Soriano (Eds.), Components of emotional meaning: A Sourcebook (pp. 106-125). Oxford: Oxford University Press. https://doi.org/10.1093/acprof:oso/9780199592746.003.0008

Fontaine, J. R., \& Scherer, K. R. (2013b). From emotion to feeling: The internal structure of the feeling component. In J. R. Fontaine, K. R. Scherer \& C. Soriano (Eds.), Components of emotional meaning: A Sourcebook (pp. 129-148). Oxford: Oxford University Press. https://doi.org/10.1093/acprof:oso/9780199592746.003.0009

Fontaine, J. R., Scherer, K. R., \& Soriano, C. (2013). The why, the what, and the how of the GRID instrument. In J. R. Fontaine, K. R. Scherer \& C. Soriano (Eds.), Components of emotional meaning: A Sourcebook (pp. 83-97). Oxford: Oxford University Press. https://doi.org/10.1093/acprof:oso/9780199592746.003.0006 
Geeraerts, D. (2010). Theories of Lexical Semantics. Oxford: Oxford University Press. https://doi.org/10.1093/acprof:oso/9780198700302.001.0001

Izdebska, W. D. (2015). Semantic field of ANGER in Old English. Doctoral dissertation. Retrieved from http://theses.gla.ac.uk/6227/

Kachru, B. (2006). English: World Englishes. Encyclopedia of Language \& Linguistics, 195-202. https://doi.org/10.1016/b0-08-044854-2/00645-3

Karakaş, A. (2017). English voices in 'Text-to-speech tools': Representation of English users and their varieties from a world Englishes perspective. Advances in Language and Literary Studies, 8(5), 108-119. https://doi.org/10.7575/aiac.alls.v.8n.5p.108

Kim, E. (2015). Diachronic and Synchronic Properties of English Prepositions in Emotion Constructions: From a Grammaticalization Perspective. Doctoral dissertation. Seoul: Hankuk University of Foreign Studies.

Kim, E. (2016). Causality-Encoding of at and by in Emotion Constructions in English. Australian Journal of Linguistics, 37(1), 1-18. https://doi.org/10.1080/07268602.2016.1169974

Kim E. (2018). Persistence of spatial meanings in the conceptualization of causality: at, by, with and about in emotion constructions. Poznan Studies in Contemporary Linguistics, 54(2), 223-254. https://doi.org/10.1515/psicl-2018-0009.

Kövecses, Z. (2000a). Metaphor and emotion: Language, culture and body in human feeling. Cambridge: Cambridge University Press.

Kövecses, Z. (2000b). The concept of anger: Universal or culture specific? Psychopathology, 33, 159-170. https://doi.org/10.1159/000029139

Ku, Y. (2019). A Pragmatic Approach to English Emotion [Adjective + Preposition] Collocations. Master's thesis, Korea University, Seoul.

Li, D. (2016). Collocation Extraction and the Collocational Features of Verbs in China English. Master's thesis, Dalian University of Technology, China.

Nelson, G. (1996). The design of the corpus. In S. Greenbaum (Ed.), Comparing English worldwide: The International Corpus of English (pp. 27-35). Oxford: Clarendon Press.

Oatley, K., \& Johnson-laird, P. N. (1987). Towards a Cognitive Theory of Emotions. Cognition \& Emotion, 1(1), 29-50. https://doi.org/10.1080/02699938708408362

Osmond, M. (1997). The prepositions we use in the construal of emotion: Why do we say fed up with but sick and tired of? In S. Niemeier \& R. Dirven (Eds.), The language of emotions: Conceptualization, expression, and theoretical foundation (pp. 111-133). Amsterdam: John Benjamins. https://doi.org/10.1075/z.85.09osm

Rong, H. (2007). A Corpus-based Study of the Use of Emotion Vocabulary by Chinese EFL Learners. Teaching English in China, 6, 114-121, 128. https://doi.org/10.1088/1126-6708/2007/06/014

Schmid, H. (1994). Tree Tagger (Version 3.0). Stuttgart, Germany: University of Stuttgart. Retrieved from https://www.cis.uni-muenchen.de/ schmid/tools/TreeTagger/\#Windows.

Sorial, S. (2017). The Expression of Anger in the Public Sphere. Journal of Social Philosophy, 48(2), 121-143. https://doi.org/10.1111/josp.12188.

Tsai, J. L., Simeonova, D. I., \& Watanabe, J. T. (2004). Somatic and Social: Chinese Americans Talk about Emotion. Personality and Social Psychology Bulletin, 30(9), 1226-1238. https://doi.org/10.1177/0146167204264014.

Van, K. G. A., \& Côté, S. (2007). Expressing anger in conflict: When it helps and when it hurts. Journal of Applied Psychology, 92, 1557-1569. https://doi.org/10.1037/0021-9010.92.6.1557.

Wierzbicka, A. (1999). Emotions across Languages and Cultures: Diversity and Universals. Cambridge: Cambridge University Press. https://doi.org/10.1017/CBO9780511521256.

Wierzbicka, A., \& Harkins, J. (2001). Introduction. In J. Harkins \& A. Wierzbicka (Eds.), Emotions in Crosslinguistic Perspective (pp. 1-34). Berlin: Mouton De Gruyter. https://doi.org/10.1515/9783110880168.1

Woon, Y. H. J. (2009). The language of anger in Chinese and English narratives. International Journal of Bilingualism, 13(4), 481-500. https://doi.org/10.1177/1367006908353235 


\section{Copyrights}

Copyright for this article is retained by the author, with first publication rights granted to the journal.

This is an open-access article distributed under the terms and conditions of the Creative Commons Attribution license (http://creativecommons.org/licenses/by/4.0/). 